\title{
L'analyse d'un dispositif de langues à travers des "carnets de bord" des apprenants de niveau A2a
}

\section{OpenEdition}

Journals

Édition électronique

URL : http://journals.openedition.org/rdlc/2091

DOI : 10.4000/rdlc.2091

ISSN : 1958-5772

Éditeur

ACEDLE

Référence électronique

Nicola Macré, «L'analyse d'un dispositif de langues à travers des "carnets de bord" des apprenants de niveau A2a », Recherches en didactique des langues et des cultures [En ligne], 6-1 | 2009, mis en ligne le 21 août 2017, consulté le 24 avril 2019. URL : http://journals.openedition.org/rdlc/2091 ; DOI :

$10.4000 /$ rdlc. 2091

Ce document a été généré automatiquement le 24 avril 2019

\section{cc) (†)}

Recherches en didactique des langues et des cultures is licensed under a Creative Commons AttributionNonCommercial-NoDerivatives 4.0 International License 


\title{
L'analyse d'un dispositif de langues à travers des "carnets de bord" des apprenants de niveau A2a
}

\author{
Nicola Macré
}

\section{Introduction}

1 Marginalisé dans les années soixante-dix, l'apprentissage des langues en autoformation est aujourd'hui largement adopté par les institutions supérieures. Répondant autant à des besoins pédagogiques qu'économiques et technologiques avec le développement des Technologies de l'Information et de la Communication pour l'Éducation (Tice), ces dispositifs d'autoformation peuvent offrir des moyens efficaces de se former dans un environnement sans cesse plus complexe et changeant.

2 Néanmoins, des difficultés de mise en œuvre des pratiques pédagogiques visant à l'autonomie ont été relevées dans diverses études (Albero, 2003). Celles-ci soulignent également la complexité des changements à entreprendre afin que l'objectif d'autoformation soit effectivement réalisable (Barbot, 2003). La prise en compte de l'apprenant est devenue inhérente au système éducatif. C'est un facteur déterminant pour un enseignement efficace et autonomisant.

3 En 2000, l'intégration des apprenants Lansad ${ }^{1}$ dans l'enseignement supérieur s'est traduite par une augmentation du nombre d'étudiants de niveau $\mathrm{A}^{2}$ dans des centres de langues destinés à l'origine aux étudiants de niveaux B2 ou C1 en anglais.

4 Afin d'appréhender les besoins spécifiques de ces apprenants, cet article propose, par le biais de l'analyse qualitative d'un corpus de carnets de bord de douze apprenants de niveau A2, d'identifier un certain nombre de variables linguistiques, psychologiques, cognitives, sociales et culturelles, afin d'établir la manière dont elles interfèrent dans un processus d'appropriation ${ }^{3}$. 
5 Nous détaillerons d'abord le contexte de la recherche ainsi que la méthodologie mise en œuvre pour répondre aux questions exploratoires suivantes: comment le processus d'autonomisation intervient-il dans cet apprentissage? Quels sont les indicateurs d'autonomisation? Quels facteurs peuvent bloquer ou favoriser le processus? Des exemples concrets saisis à partir du corpus nous permettront ensuite d'expliciter et de comparer les parcours suivis, en mettant en évidence quelques indicateurs révélateurs d'un processus d'appropriation.

\section{Méthodologie}

\subsection{Le contexte}

Dans le cadre de l'apprentissage de l'anglais en autoformation accompagnée à l'université Lyon 2, les apprenants de niveau peu avancé sont souvent invités à prendre en charge leur formation au même titre que les apprenants de niveaux avancés. Pourtant ils doivent faire face à davantage d'obstacles que ces derniers, autant psychologiques que cognitifs, et combler des écarts de connaissance plus importants.

7 Enseignante auprès d'un groupe d'étudiants de niveau A1 et A2 en anglais, nous avons voulu d'abord et surtout encourager une réflexion sur l'apprentissage et ensuite mieux déterminer plus précisément les difficultés rencontrées par ces étudiants pendant leur travail en autonomie et pendant les cours.

\subsection{Le programme mis en place}

Les cours/tutorats ont été divisés en deux parties. La première (le tutorat) a été consacrée au travail personnel, l'objectif étant d'encourager des échanges et le partage d'information parmi les apprenants, de les aider à se connaître, à reconnaître leurs besoins, à analyser leurs attitudes face à l'apprentissage de l'anglais, à fixer les objectifs, et à s'auto évaluer.

Une seconde partie (cours) a été consacrée au développement des compétences linguistiques et l'accent a été mis sur la production orale. Pour aboutir à celle-ci, les sources suivantes ont été exploitées : un texte à lire, un enregistrement audio ou une vidéo à comprendre ont permis un travail de réflexion au niveau des stratégies à mettre en place.

10 Le travail demandé était de tenir un journal et de rendre trois tâches ${ }^{4}$ à des dates spécifiques dont deux au choix en consultation avec le tuteur. L'évaluation a compris un test de mi-semestre et une évaluation finale qui constituaient chacun respectivement 10 et $30 \%$ de la note. Le travail en autonomie et les tâches ont constitué $60 \%$ de la note. Le journal lui-même n'a pas fait l'objet d'une notation.

\subsection{Le journal}

11 Outil de recherche particulièrement adapté à des enquêtes sur le comportement dans son contexte, le journal a été retenu comme moyen pertinent de recherche pour ses qualités " d'outil d'introspection" (Nunan, 1992: 118) et pour son format d'enregistrement d'un processus qui évolue au fil du temps : 
Indeed the theme of change over time and the sense of writing about a process is one that resonates directly with the use of diaries in educational research (McDonough \& McDonough, 1997: 121).

\subsection{Les consignes}

Dès le premier cours, les étudiants ont été invités à tenir un journal d'une huitaine de pages, rédigé en français (cf. annexe 1 pour les détails des consignes).

\subsection{Corpus}

\subsubsection{Présentation du corpus et de la démarche d'analyse}

13 La population est composée de douze apprenants non spécialistes de langue en troisième année d'université ayant choisi l'anglais UE libre et dont le nombre d'années d'anglais antérieur varie entre un et sept ans. Il y a onze sujets féminins pour un masculin issus d'un groupe de trente-cinq apprenants. Cependant, la proportion d'étudiants étrangers, français et adultes ${ }^{5}$ est égale.

14 À titre exploratoire, nous avons choisi les apprenants selon leur niveau de réflexion dans les journaux et la variété des difficultés qu'ils avaient à surmonter: en tant qu'enseignante, nous avions connaissance des progrès obtenus dans les tâches et les parcours suivis par les apprenants.

\subsubsection{Définition de carnet /journal de bord}

15 Le journal est peu connu des apprenants et souvent confondu avec d'autres termes : carnet de bord, logbook et diary. À la suite de Guichon, l'analyse du corpus a également donné l'occasion de distinguer trois genres écrits: le carnet de bord, qui correspond à un simple compte-rendu des événements, le journal intime, qui décrit les évènements mais qui est à dominance émotionnelle et le "récit de formation" qui regroupe les deux précédents dans une réelle analyse des actions entreprises : description et émotions (Guichon, $2007: 4$ ). Les journaux sélectionnés répondent à la troisième définition.

\section{Les démarches d'analyse}

\subsection{L'autonomie, concept ou processus?}

16 La notion d'autonomie est très complexe et difficile à mesurer. Sa manifestation peut varier selon l'âge, le niveau de compétence et le contexte d'apprentissage (Little, 1991 : 4). En tant que "capacité"6, l'apprenant peut posséder le savoir-faire, mais manquer d'envie et de motivation pour accepter la responsabilité de ses décisions.

17 Cependant, bien que l'autonomie dans l'apprentissage semble difficile à cerner à partir des définitions globales, nous pouvons constater qu'il ne s'agit pas d'une finalité ou d'un comportement à relever, mais plutôt "d'un moteur interne" (Barbot \& Camatarri, 1999: 8) et d'un processus dynamique et continu ("action indépendante", Little, 1991:49). Ce dernier se caractérise par un besoin naturel de l'apprenant de construire du sens en tenant compte de ses besoins, de ses capacités ("selon le mode d'être qui lui est propre et ses 
nécessités", Barbot, $2000: 2$ ), de ses motivations ("en prenant lui-même les décisions concernant les objectifs à atteindre", Holec, cité par Gremmo dans Albero, $2003: 155)$ et de son environnement ("le sujet élabore ses propres lois", Hoffmans-Gosset, 2000 : 102).

En considérant les diverses définitions concernant l'autonomie, nous remarquons également l'importance accordée à certains facteurs psychologiques et cognitifs dans le contrôle de l'apprentissage. Selon Linard (2003 : 247), "l'autonomie est une capacité de haut niveau, cognitive, mais aussi psychologique et sociale". L'attention, l'autocontrôle, et la confiance en soi apparaissent ainsi comme des qualités nécessaires pour "réaliser et élaborer" un apprentissage en autonomie (ibid.), (voir également Barbot, $2000: 22$; Holec, cité par Gremmo dans Albero, $2003: 155$; Little, 1991 : 49).

En effet, après une lecture flottante des journaux, ce ne sont pas des comportements isolés et individuels qui sont apparus, mais un ensemble de caractéristiques et de capacités qui influencent et déterminent l'interaction entre l'apprenant et son environnement de façon cyclique.

\subsection{Comment le processus d'autonomisation intervient-il dans l'apprentissage?}

Dans l'apprentissage des langues, les processus en question s'élaborent en fonction des actions entreprises pour développer les compétences en langue. Dans le cadre de la formation, les apprenants ont dû rendre trois tâches ${ }^{7}$ et travailler sur des objectifs individuels établis en début de formation. En identifiant le type d'action et sa motivation, il est possible d'identifier les facteurs susceptibles de dynamiser le processus, c'est-à-dire les capacités sociales, cognitives et affectives qui permettent à l'apprenant de se prendre en charge et de contrôler (évaluer, autoréguler) son apprentissage selon ses besoins et les exigences de l'environnement. En comparant les deux tableaux (cf. figure $1 \mathrm{~A}$ et figure 1B), nous constatons que l'absence de réflexion et de métaréflexion empêche une régularisation et une réactivation du processus : 
Figure 1A - Processus sans réactivation.

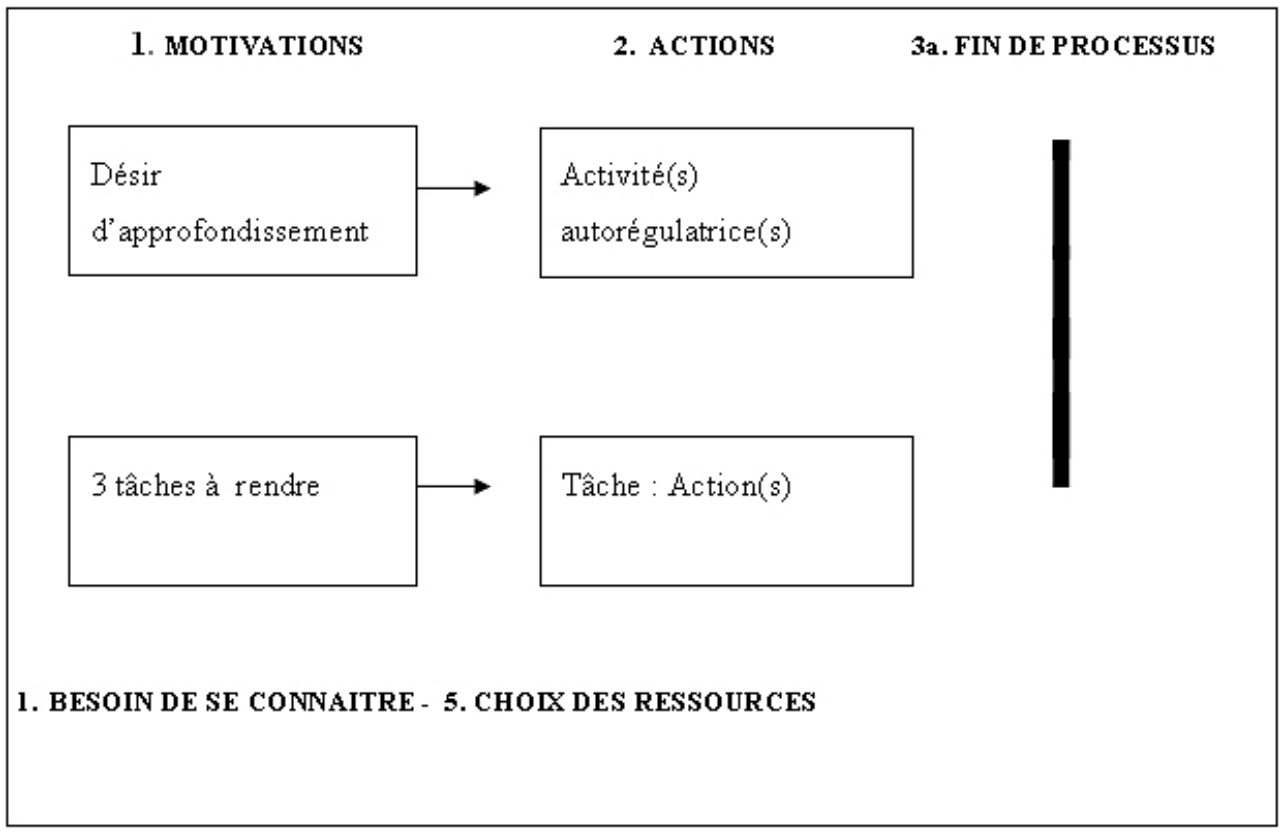

Figure 1B - Processus avec réactivation.

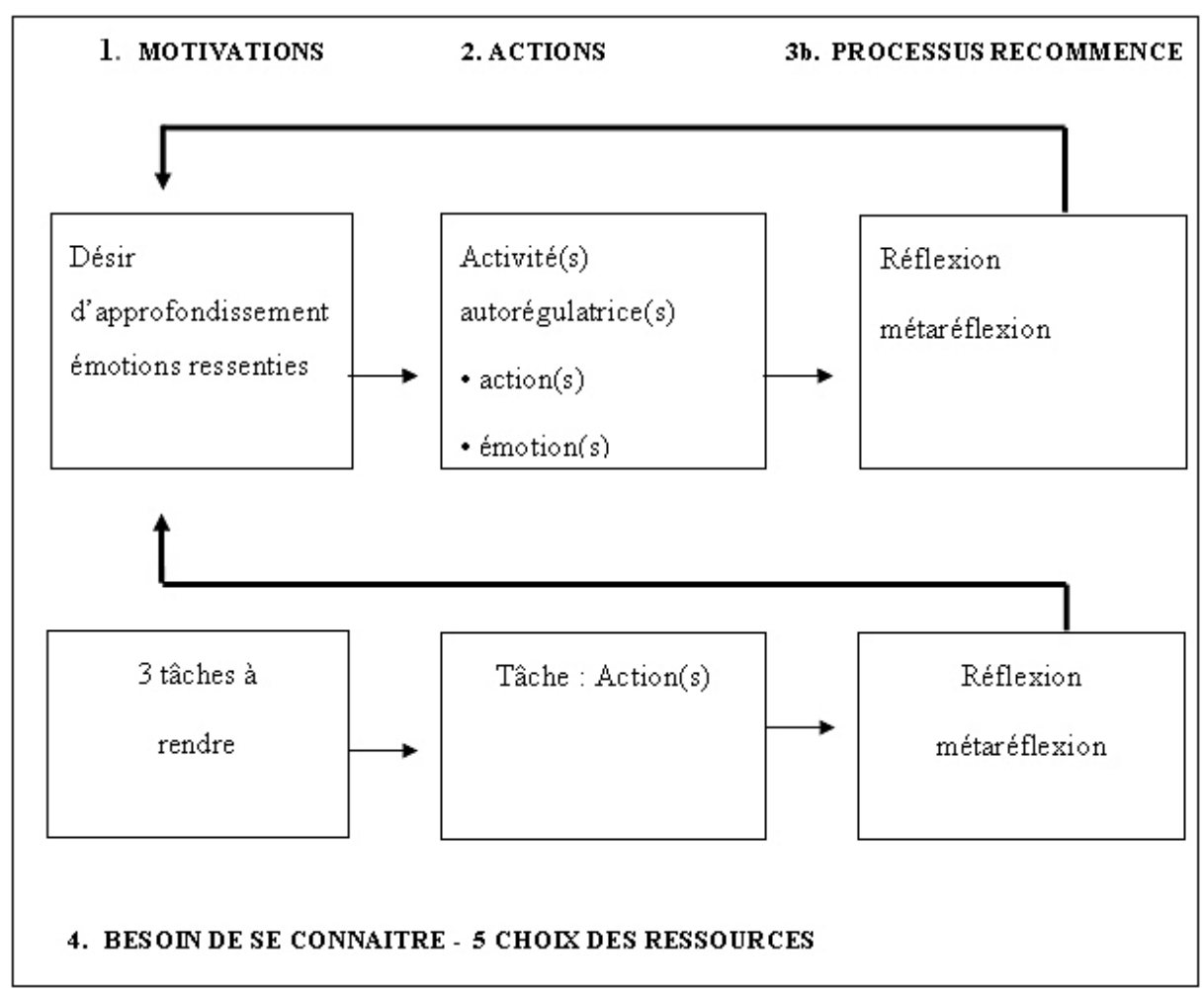

21 1. Avant de mettre en place une action, l'apprenant doit être motivé. La motivation de l'activité régulatrice et l'action nécessitent des déclencheurs qui sont externes à la personne (une note ou les consignes du tuteur) ou internes (une difficulté que l'apprenant a remarquée ou ressentie) (cf. figures 1A et 1B). 

d'établir des indicateurs d'autonomisation caractéristiques de l'autoformation en langue accompagnée. En adoptant les cinq concepts-clés de l'étude qui sont traduits en termes de compétences à acquérir chez l'individu, nous proposons les indicateurs suivants :

Figure 2 - Grille d'analyse adaptée de N. Tremblay (2003).

\begin{tabular}{|l|l|}
\hline 1. & $\begin{array}{l}\text { Apprendre à apprendre : métaapprentissage (l'apprenant se connaît-il et } \\
\text { apprend-t-il à se connaître ?) }\end{array}$ \\
\hline 2. & $\begin{array}{l}\text { Apprendre en action : praxis (l'apprenant modifie les actions mises en place } \\
\text { selon son parcours) }\end{array}$ \\
\hline 3. & $\begin{array}{l}\text { Apprendre en mouvance (avec souplesse) : cadre organisateur qui s'ajuste } \\
\text { selon les contraintes de l'environnement }\end{array}$ \\
\hline 4. & Apprendre en interaction : réseautage (ressources sociales) \\
\hline 5. & Apprendre en création : stochastique (aléatoire, tolère l'incertitude) \\
\hline
\end{tabular}




\section{Résultats : pour quelle raison le processus peut-il être bloqué ou favorisé?} exprimés au début de la formation dans les journaux restent pour l'essentiel de l'ordre de "je suis timide", "je n'ose pas parler", "j'essaie de tout comprendre" et "je veux traduire d'abord". Les apprenants adultes semblent se connaître davantage et tiennent compte de cette information dès le début de la formation ${ }^{9}$ :

Extrait $1:$ FRE2

Après une première année en débutant 1 où j'ai pu apprendre certaine bases, j'ai donc pu définir un premier problème, celui de la compréhension globale. Extrait 2 : SEL2

À cette étape de mon retour à l'anglais j'ai envie de progresser en affrontant, corrigeant mes erreurs. Certains apprenants se bloquent devant leurs erreurs. Moi, je les considère comme faisant partie de mon apprentissage et comme très utile pour progresser. J'ai besoin d'y être confronté pour les maîtriser. Je voudrais avoir le temps de me poser des questions sur ce que je dis, la manière de le dire. Je voudrais être corrigée pour progresser. J'ai soif de construction...

Dans ces extraits, les deux apprenants, forts de leurs expériences passées, réfléchissent sur leurs besoins de façon positive. Ils semblent prêts à "affronter" et à "confronter" leurs difficultés, à corriger et à "définir" un parcours pour "construire" et "progresser" dans leurs apprentissages respectifs. 


\subsection{Se connaître : pendant la formation}

34 À partir du moment où l'apprenant se rend compte de l'origine de ses difficultés et est prêt à modifier ses stratégies, il semble apprendre à se connaître au fur et à mesure que la formation avance. Dans les exemples suivants, CLA1 apprend qu'elle est plus motivée et plus efficace si le travail est utile et donc intéressant tandis que FRE2 apprend qu'elle a un problème de compréhension orale à la vitesse normale et qu'il faut qu'elle puisse s'y habituer doucement. Les deux apprenants modifient leur apprentissage en fonction de leurs besoins.

Nous pouvons constater le schéma suivant :

- travail avec un mode d'apprentissage connu : regarder un film et utiliser une méthode ;

- constat des problèmes à partir des évaluations personnelles, "efficacité", "intéressant" ;

- activité régulatrice avec constat des erreurs à éviter à l'avenir, "n'étant rattaché à aucune autre démarche" et des points positifs de l'expérience, "cela m'a permis de développer néanmoins l'idée d'un travail pluriel autour d'un thème".

Extrait 3 : CLA1

Les premiers temps ne sachant pas comment m'y prendre, j'ai travaillé chez moi avec une méthode que j'ai acheté, English plus, niveau débutant 1 et 2 . J'ai alors travaillé le thème des saisons et de l'agence de voyage ayant déjà l'objectif d'utiliser mon travail pour une visite en Angleterre. Or, n'étant rattaché à aucune autre démarche, ce travail ne sur à mon avis pas très efficace. Cela m'a permis de développer néanmoins l'idée d'un travail pluriel autour d'un thème.

J'ai donc commencé par deux séances en salle de documentation où j'ai trouvé un livre intéressant avec des exercices de grammaire et de vocabulaire.

Extrait 4 : FRE2

Le film n'a pas été sans problème. J'ai donc dû a un moment travailler sur le débit du langage avec un autre CD rom "Business in London " qui offrait l'avantage de régler en temps réel le débit paroles.

\subsection{Réfléchir dans et sur l'action : l'importance de la réflexion et de la métaréflexion pour faire évoluer l'apprentissage}

L'apprentissage s'effectue à partir de réflexions sur l'action. La réflexion et métaréflexion permettent aux apprenants d'assimiler et d'accommoder (Piaget, 1966) ${ }^{10} \mathrm{l}^{\prime}$ information pour faire évoluer l'apprentissage. Dans l'extrait suivant (extrait 5), nous pouvons constater comment l'apprenant, en réfléchissant sur les activités du cours/tutorat, arrive à accepter sa part de responsabilité dans le travail à fournir et à comprendre l'utilité du tutorat "boîte à outil" pour ces propres recherches. Tout d'abord en comparant le cours/ tutorat à un cours traditionnel, il explique son étonnement. Ses représentations d'un cours traditionnel sont bouleversées. Pour assimiler l'information et modifier ses représentations, l'apprenant réfléchit sur les raisons de cette nouvelle structure de cours. On peut remarquer l'utilisation répétitive de "il me semble que", qui indique le début des réflexions et marque une différence entre les deux premières phrases ponctuées de verbes conjugués à la première personne : "j'ai été étonné", "je pensais que", "je m'attendais à ". Dans les dernières phrases (voir Figure 3 ci-dessous), l'apprenant bascule vers une compréhension et une acceptation d'une structure qui implique davantage 
d'investissement de sa part: "Mais vous me direz on n'a rien sans rien", un proverbe qui vient à la fin pour résumer les réflexions sur le travail important à fournir. Cette dernière partie de nature "métaréflexive" est d'ailleurs mise en relief par la phrase introductive "avec le recul".

Figure 3 - Exemple de CLA1.

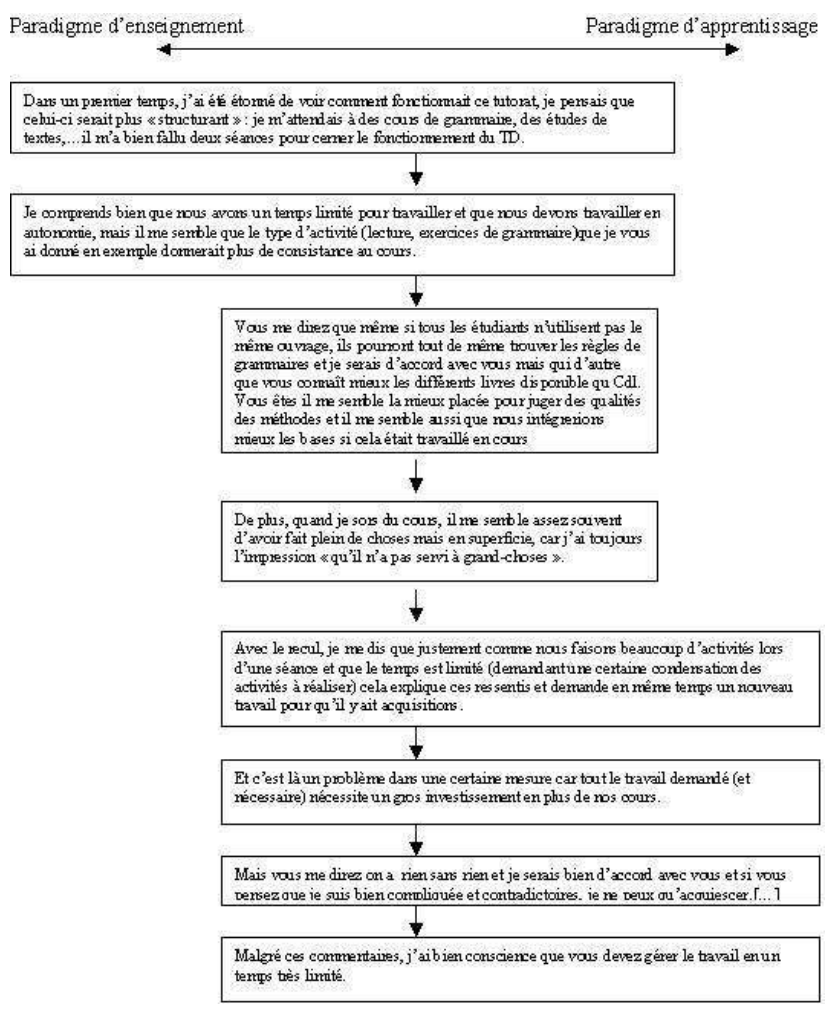

\subsection{Se connaître : représentations du cours}

Sans cette réflexion l'apprenant aurait pu rester bloqué, frustré d'un "mauvais cours traditionnel" qui ne lui aurait pas transmis les informations de la manière dont il avait l'habitude.

Dans l'exemple suivant on peut comparer l'interprétation du cours de ce même apprenant avec l'interprétation d'un autre étudiant. Bien que ce soit difficile à croire, ils ont suivi le même cours. CLA1 est frappé par l'écart entre ce cours et un cours traditionnel alors que KAR1 interprète les activités selon un schéma de cours traditionnel. Les deux apprenants remarquent et interprètent la séance de deux manières différentes :

\section{Extrait 5 : CLA1}

Dans un premier temps, j'ai été étonné de voir comment fonctionnait ce tutorat, je pensais que celui-ci serait plus "structurant ": je m'attendais à des cours de grammaire, des études de textes,...il m'a bien fallu deux séances pour cerner le fonctionnement du TD.

Extrait $6:$ KAR1

Lors de cette séance, nous avons vu les temps(tenses): present simple/ present continuous, past future. Je ne saisis pas la différence entre les deux présents. 


\subsection{Se connaître : actualisation des pratiques antérieures}

\section{représentations entre le début et la fin de la formation. Il renoue avec ses pratiques} antérieures du lycée : dictionnaire, exercices de grammaire et devoirs à rendre :

Extrait 7 : CHA1 début de formation

J'avais rendu mon premier devoir rédigé, on devait décrire les membres de notre groupe. J'avais du mal à mettre toutes mes idées et informations en ordre. Tout ceci ne s'est pas fait sans difficulté. Il m'a fallu consulter le dictionnaire, le livre de grammaire, de conjugaison pour améliorer mes connaissances.

Extrait 8 : CHA1 vers la fin de la formation

Il y avait un travail de correction sur nos devoirs de plus il était important

d'avoir préparé tous les exercices demandés pour les cours prochains.

\subsection{Se connaître : l'importance d'un déclencheur}

Sur les douze apprenants, huit soulignent un événement "déclencheur" qui leur a permis de changer de pratique. Nous pouvons noter que ces moments de prise de conscience sont souvent caractérisés par une situation linguistique que l'apprenant pensait déjà maîtriser. Le cours sur la prononciation de l'alphabet est souvent donné en exemple. Bien que les émotions ressenties soient "désagréables", il semble que cet évènement soit nécessaire pour faire réagir l'apprenant et déclencher le processus d'autonomisation.

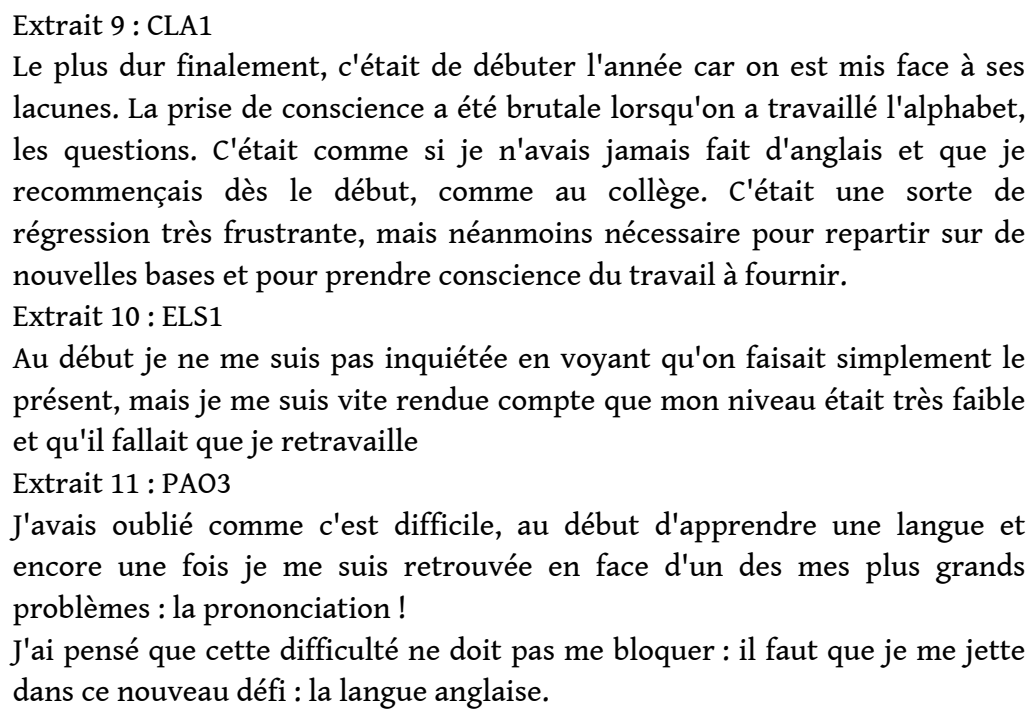

Sans cet évènement "déclencheur", les pratiques des apprenants indiquent une " actualisation de pratiques antérieures" (Paquelin \& Choplin, $2003: 183$ ), ce que nous avons pu constater plus haut (cf. exemples de KAR1 et de CHA1). Les activités peuvent être plus ou moins nombreuses, c'est la note ou les consignes du tuteur qui en sont à l'origine. L'apprentissage et le choix des ressources restent superficiels et identiques aux devoirs du collège ou du lycée. Dans l'extrait 12, malgré le fait que l'apprenant, HOU3, ait déjà reçu l'évaluation de sa première tâche (des conseils pour corriger ses erreurs et améliorer 
ses résultats), il ne modifie pas sa façon de travailler et reste "enfermé" dans ses pratiques antérieures :

Extrait $12:$ HOU3

Pour faire cet exercice je me suis servie des documents donnés par le professeur, sur la forme interrogatrice, en plus de ça j'ai utilisé le dictionnaire pour traduire quelques mots et des fois des expressions. Le travail était très long, les mots chercher dans le dictionnaire : "next, party, Saturaday, flat to clean, after, room ; bedroom, dining room, coats, food snacks", pour faire cet exercice j'ai mis au moins deux heures, comme j'ai fait les exercices dans les polycopies données par le professeur.

\subsection{L'action : réflexion, métaréflexion et autorégulations} de poser des questions aux personnes ressources du dispositif qui ne sont pas concrétisées.

\begin{abstract}
Extrait 14 : CLA1 Lors de sa première visite dans la salle de documentation En réfléchissant, avec un peu de recul, je me suis rendue compte que cela était du à une mauvaise organisation; je demandais plus aux personnes qui m'avaient déjà un peu guidé (vous et les personnes accueillants les étudiants) et celles-ci n'étaient pas disponibles pour aider chaque étudiant, à commencer l'apprentissage des utilisations des outils. Résignée et frustrée devant ce fonctionnement, (se débrouiller après une petite introduction au $\mathrm{CDL}$ ), je me suis organisée pour essayer de venir travailler régulièrement.
\end{abstract}

Extrait 13 : DEN2 rendu la tâche 2 sans connaître le CDL, cela effraie un peu d'autant plus que des copies papier, de cassettes vont être laissées, le CDL n'ouvrira que le 17/10. Tout cela s'emmêle, je verrai plus clair sur le terrain. De toute façon, j'ai une langue pour poser des questions. À titre indicatif, la comptabilisation des actions et des réflexions permet de souligner des tendances qui sont vérifiées ultérieurement. son apprentissage (métaréflexion) semble ne pas ressentir le besoin de déclencher une activité régulatrice. Par ailleurs, un apprenant qui réfléchit beaucoup sur son apprentissage mais ne le régularise pas par des actions ne fera pas évoluer ses pratiques. L'apprenant DEN2, un apprenant qui ne fait pas beaucoup de progrès dans son apprentissage, met en place six actions et effectue vingt-quatre réflexions et métaréflexions alors qu'un autre apprenant, CLA1, qui progresse de façon régulière, met en place sept actions et fait six réflexions et métaréflexions :

45

Sans réflexion et métaréflexion, l'apprenant peut rester bloqué dans un processus centré sur l'enseignement comme nous l'avons vu précédemment (cf. HOU3). En effet, un étudiant qui a mis en place sept actions et aucune réflexion ou métaréflexion peut difficilement modifier sa façon d'apprendre. 

personne qui déclenche la réflexion, semblent primordiaux dans un processus d'autonomisation. Bien que les conseils de l'enseignante aient leur importance, l'objectif du processus d'autonomisation est que l'apprenant apprenne à s'auto évaluer et à mettre en place ses propres activités.

\subsection{Tolérer l'incertitude}

Habitués à des formations linéaires, l'adaptation au nouveau mode aléatoire d'apprentissage s'avère difficile pour les apprenants. Avec le recul, tous les apprenants se rendent à l'évidence qu'il leur faut développer une capacité d'organisation, et un certain nombre le démontre à la mi-parcours. Les apprenants qui semblent le moins bien tolérer l'incertitude sont les adultes.

Des imprévus (problèmes techniques ou difficultés à atteindre les objectifs fixés au début de la formation) se sont traduits en obstacles insurmontables. Il a fallu une semaine de repos à un apprenant peu habitué à modifier ses plans pour y faire face :

Extrait 15 : ELI2

Mes difficultés d'organisation par rapport aux problèmes du CDL m'ont beaucoup perturbée, j'accuse le contre coup. Analyste programmeur en informatique de formation, je suis habituellement très organisée et je me sens mieux lorsque je peux bien prévoir au moins à moyen terme.

\subsection{Les obstacles émotionnels à surmonter}

C'est le sentiment d'un immense travail à accomplir en très peu de temps qui a prédominé.

Extrait 16 : CLA1

Le travail me paraissait énorme, que je ne savais pas par où commencer, et que c'est grâce au tutorat que j'ai pu m'accrocher

Tous les apprenants sans exception ont ressenti une frustration liée à leur faible niveau d'anglais et à l'énorme écart qu'ils devaient combler.

Extrait 17 : CLA1

C'est comme si j'avais une gigantesque montagne devant moi mais que je ne savais pas comment m'y prendre pour monter tout en haut. Il m'a donc fallu me dépasser ou plutôt dépasser mes angoisses pour accepter aussi de rendre un travail simple. si je n'avais jamais fait d'anglais et que je recommençais dès le début, comme au collège". Pour la plupart, ce sentiment s'est estompé avec l'aide fournie par le tutorat. Néanmoins, sans régulation, le processus d'autonomisation et d'apprentissage peut définitivement s'arrêter.

En relevant les frustrations liées aux compétences linguistiques, nous avons pu noter qu'un grand nombre d'entre elles concerne les difficultés liées à la mémorisation et à la réutilisation orale du vocabulaire : 
Extrait $18:$ KAR1

Ma seule déception et grosse difficulté s'est de parler anglais. Les mots ne me viennent pas, j'hésite, je me bloque. Je n'arrive à construire que des phrases très simples et je mets énormément de temps pour trouver mes phrases.

Extrait 19: TET2

J'ai des problèmes avec le vocabulaire. Je n'arrive pas le retenir longtemps. Parfois, je sais que j'ai appris ce mot, mais je ne me rappelle pas.

Extrait 20 PAO3

Je me sens un peu bloqué quand je parle devant les autres et c'est bizarre parce qu'en français tout ça n'arrive pas.

Le travail en groupe a paru encourager les apprenants dits "timides", car l'entraide des autres membres du groupe les a mis en confiance.

Extrait 21 : ELS1

Je suis intimidée à l'idée de parler seule à l'orale devant une classe. Or, le système établi dans ce cours me permet vraiment de participer car je savais qu je devrais parler devant la classe, mais cette année, nous fonctionnons en petit groupes de trois ou quatre personnes, ce qui m'a vraiment mise à l'aise et permis de prendre la parole autant que les autres.

Cependant, d'après les journaux, le traitement de l'erreur et les progrès qu'ils ont pu faire en groupe ne semblent pas acquis chez tous les apprenants (surtout chez les adultes) :

Extrait 22 : DEN2

Personnellement, je ne me sens pas assez mûr pour aider quelqu'un à progresser

Extrait 23 : TET3

Si on parle entre nous, mais ce n'est pas pareil, parce qu'on parle avec des

fautes. Personne ne corrige.

Extrait 24 : CLA1

Je trouve que nous avons suffisamment de temps pour travailler l'oral entre nous mais je ne suis pas sûr qu'il soit suffisant pour développer notre expression. comme nous avons pu le souligner, une modification du comportement lié à l'apprentissage qui demeure étrangère aux apprenants français et étrangers ayant pris part à l'enquête.

Bien que l'apprenant soit responsable de son apprentissage, il peut avoir besoin d'intermédiaire pour l'aider à débloquer ou à faciliter le processus d'autonomisation. Les connaissances de son propre profil, de ses attitudes et de ses besoins semblent indispensables chez ces apprenants afin de faciliter l'activation du processus. Cependant, sans la réflexion et la métaréflexion qui permettent la mise en place d'actions 
régulatrices qui à leur tour maintiennent le système d'apprentissage en équilibre, l'apprentissage peut rester bloqué.

Dans des cours de trente-cinq étudiants souvent confiés à des enseignants vacataires peu habitués à la formation en autonomie accompagnée, comment développer la réflexion et la métaréflexion de l'apprenant? Comment concilier la certification du $\operatorname{CLES}^{11}$ et le nombre limité d'heures de cours/tutorat en présentiel tout en permettant aux apprenants de progresser en anglais alors qu'après sept ou huit ans de formation au lycée, ils sont restés à un niveau A2 ?

En identifiant les sources de ses obstacles, ce projet s'inscrit dans une visée d'élaboration des dispositifs appropriés à tous les profils d'apprenants et marque la première étape d'une étude de doctorat qui s'interroge sur le type et la forme d'aide pédagogique nécessaires aux apprenants de niveau A2.

\section{BIBLIOGRAPHIE}

Albero, B. (2003). "Autoformation et contextes institutionnels : une approche socio-historique". In Albero, B. (dir.). Autoformation et enseignement supérieur. Paris : Lavoisier.

Barbot, M.-J. \& Camatarri G. (1999). Autonomie et Apprentissage, l'innovation dans la formation. Paris : PUF.

Barbot, M.-J. (2000). Les auto-apprentissages. Paris : CLE international.

Barbot, M.-J. (2003). "Médiatisation dans l'enseignement supérieur : vers un nouveau paradigme éducatif ?". ALSIC, vol. 6, n 1.pp. 175-189. http://alsic.u-strasbg.fr

Bardin, L. (1977). L'analyse de contenu. Paris : PUF.

Barnier, G. (2001). Le tutorat dans l'enseignement et la formation. Paris: L'Harmattan.

Ellis, R. (2003). Task-based Language Learning and Teaching. Oxford: OUP.

Gremmo, M-J. (2003). "Aider l'apprenant à mieux apprendre". In Albero, B. (dir.). Autoformation et enseignement supérieur. Paris : Lavoisier.

Guichon, N. (2007). "L'identité professionnelle en construction des futurs formateurs de langue". Actes du colloque CDIUFM : "Qu'est-ce qu'une formation professionnelle universitaire des enseignants ?" Arras.

Guichon, N. (2006) Langues et TICE Méthodologie de conception multimedia. Paris : Ophrys.

Hoffmans-Gosset, M.-A. (2000). Apprendre l'autonomie. Apprendre la socialisation. Lyon : Chronique Sociale.

Holec, H. (1979). Autonomie et apprentissage des langues étrangères. Strasbourg : Conseil de la coopération culturelle du Conseil de l'Europe.

Linard, M. (2003). "Autoformation, étique et technologies : enjeux et paradoxes de l'autonomie". In Albero, B. (dir.). Autoformation et enseignement supérieur. Paris : Lavoisier.

Little, D. (1991). Learner Autonomy. Definition, Issues and Problems. Dublin: Authentik. 
Littlewood, W. T. (1996). "Autonomy: an anatomy and a framework". System, 24 n 4. pp. 427-435.

McDonough, J. \& McDonough, S. (1997). Research Methods for English Language Teachers. Londres: Arnold.

Nunan, D. (1992). Research Methods in Language Learning. Cambridge: Cambridge University Press.

Paquelin, D. \& Choplin, H. (2003). "Du prescrit au vécu : l'enjeu des régulations". In Albero, B.

(dir.). Autoformation et enseignement supérieur. Paris : Lavoisier.

Piaget, J., Inhelder B., (1966). La psychologie de l'enfant, Paris, PUF.

Tremblay, N. (2003). L'autoformation : pour apprendre autrement. Montréal : Les presses de

l'université de Montréal.

\section{ANNEXES}

\section{Annexe 1}

Dès le premier cours, les étudiants ont été invités à tenir un journal d'une huitaine de pages, rédigé en français et respectant les consignes suivantes :

- écrire sur l'utilisation et l'apprentissage de l'anglais pendant le semestre: leur participation en cours, l'organisation et le travail personnel en dehors des cours, les tâches à accomplir, les objectifs fixés et leurs rencontres en dehors des cours (les activités et les moments de questionnements) ;

- noter la date ;

- une fois le récit terminé, écrire une conclusion dans laquelle ils réfléchissent sur leurs parcours et le journal.

Pour garder une certaine liberté d'expression et de personnalisation, le style d'écriture était libre et l'autocensure réduite à son strict minimum.

\section{NOTES}

1. Langue pour Spécialistes d'Autres Disciplines.

2. Selon le Cadre Européen de Référence.

3. Les processus au niveau linguistique et de l'autonomie (cognitive, psychologique, sociale et culturelle).

4. Définition de la tâche : elle fournit un cadre à l'activité d'apprentissage, elle implique que la priorité soit donnée au sens, elle implique que l'utilisation de la langue se fasse selon des critères qui se rapprochent de la vie extrascolaire. Elle peut inclure des compétences langagières, et débouche sur un "output" clairement défini. (Ellis, R. 2003 : 9). Traduction et résumé en quatre points essentiels de Guichon, N. $2006: 54$

5. Qui reprenaient leurs études et avaient plus de 30 ans.

6. Pour Littlewood (1996: 428), contrairement à Holec, "capacité" veut dire le savoir-faire et l'envie de faire, ce qui implique le besoin d'une motivation ainsi que la confiance en soi pour réaliser son projet.

7. Cf. note 4 .

8. Ces derniers étaient tous socialement reconnus comme experts dans des domaines aussi variés que la diététique, la minéralogie, la danse, la peinture ou l'histoire. 
9. Pour les besoins de l'analyse nous avons souhaité reproduire les extraits dans leur état brut.

10. Cité par Barnier (2001: 169).

11. Certificat de Compétences en Langue de l'Enseignement Supérieur.

\section{RÉSUMÉS}

Afin d'appréhender les besoins spécifiques des étudiants spécialistes d'autres disciplines (Lansad), cet article propose, à travers l'analyse d'un corpus de carnets de bord de 12 apprenants de niveau A2, d'identifier, à titre exploratoire, un certain nombre de variables linguistiques, sociales, psychologiques et culturelles qui leur sont propres et d'établir la manière dont elles interfèrent dans un processus d'appropriation. En explicitant et en comparant les parcours suivis cette contribution propose de mettre en évidence les facteurs susceptibles de bloquer ou d'activer le processus.

In order to apprehend the specific needs of non-specialist students (Lansad), this article analyses data collected from 12 A2 students' diaries. By identifying a certain number of linguistic, social, psychological and cultural variables caracteristic of these students, this contribution aims at establishing how they work together to enable students to evolve in a self-directed learning environement and what factors can facilitate or prevent activation of the process involved.

\section{INDEX}

Mots-clés : autonomie, processus d'autonomisation, indicateurs d'autonomisation, obstacles Keywords : autonomy, self-directed learning, autonomous process, autonomisation indicators, obstacles

\section{AUTEUR}

\section{NICOLA MACRÉ}

Nicola Macré est impliquée dans l'apprentissage des langues en autoformation accompagnée depuis plusieurs années. Elle a enseigné à l'université Lyon2 au centre de langues et est en première année de doctorat sous la direction de Marie-Josée Barbot et de Jean-Paul NarcyCombes. Sa recherche porte sur le type et la forme d'aide pédagogique nécessaires aux apprenants de niveau A2.

Courriel : n.macre[at]orange.fr.

Adresse : 500 route de Charnay, 69480, Morancé, France. 